\title{
Magnetic reconnection in large-scale cosmic plasmas
}

\author{
Kazuo Makishima \\ Department of Physics, University of Tokyo, 7-3-1 Hongo, Bunkyo-ku, Tokyo 113-0033, Japan
}

(Received July 7, 2000; Revised September 29, 2000; Accepted October 23, 2000)

\begin{abstract}
X-ray emitting hot plasmas of a temperature $2-12 \mathrm{keV}$, associated with galaxy clusters, constitute the most dominant form of baryons in the universe. The plasma is thought to be magnetized at least to $0.1-1 \mathrm{nT}$, although the $\beta$ value is relatively high at $10-100$. The plasma is confined by gravity of each cluster in a stable manner, but the member galaxies are moving through it with an approximately transonic speed. Therefore, the plasma must be in a magnetohydrodynamically turbulent condition, where frequent magnetic reconnection is expected to take place. Mainly based on observations with the $A S C A$ X-ray Observatory, evidence for such magnetic reconnection is accumulating.
\end{abstract}

\section{Introduction}

The matter in the universe is found mostly in the form of plasmas. Accordingly, the plasma-physics aspects have been developed extensively in planetary and inter-planetary disciplines, as well as solar coronal studies. Nevertheless, attempts to describe larger astronomical systems in the light of plasma physics have been surprisingly scarce. This is probably because various plasma effects (e.g., detailed configuration of magnetic fields) are difficult to detect via astronomical remote-sensing techniques. In fact, the nature of the magnetic solar corona had not been fully recognized until fine-resolution X-ray images of solar coronae became available with Skylab, Yohkoh, and SOHO.

This paper presents a novel attempt to describe the largest astronomical objects, clusters of galaxies, under a full magnetohydrodynamic (MHD) viewpoint. Filled with hot tenuous plasmas, these objects are thought to provide an ideal and fascinating laboratory to study magnetoplasma effects.

\section{Clusters of Galaxies}

A galaxy is a system formed by $\sim 10^{11}$ stars, each like the sun, and some amount of diffuse matter. The stars are held together by gravity, which is produced not only by the stars themselves but also by dark matter (DM), of which the nature is still unclear. The DM presumably forms a large halo surrounding the optically visible galaxy.

Hundreds of galaxies can also be bound together by gravity, to form an astronomical system of a higher hierarchy, called a cluster of galaxies (or a galaxy cluster). There are more than $10^{4}$ catalogued clusters in the sky. A galaxy cluster is primarily identified in the visible light as a grouping of galaxies over a narrow spatial volume, so that its primary constituent is naturally thought to be stellar component. In reality, however, there are two more dominant components of galaxy clusters.

Copy right(C) The Society of Geomagnetism and Earth, Planetary and Space Sciences (SGEPSS); The Seismological Society of Japan; The Volcanological Society of Japan; The Geodetic Society of Japan; The Japanese Society for Planetary Sciences.
One is tenuous hot plasma, called intracluster medium (ICM), compressed and confined by gravity. It permeates the cluster volume and emits bright diffuse $\mathrm{X}$-rays, including thermal Bremsstrahlung continua and emission lines from heavy ions. Figure 1 gives a comparison of the optical and $\mathrm{X}$ ray images of a cluster. Surprisingly, the spatially-integrated mass of the ICM is comparable to, or often a few times higher than, that of the stars integrated over all member galaxies. Therefore, the ICM provides the most dominant known form of baryons in the universe.

The other is again the DM, which actually dominates the total mass in each cluster. Its presence can be inferred because the total gravitating mass $M_{\mathrm{G}}$ necessary to bound the hot plasma much exceeds the estimated baryonic mass (sum of the stellar and the plasma components). Specifically, by assuming the thermal ICM pressure to balance the gravity, we can estimate $M_{\mathrm{G}}$ within a 3-dimensional radius $R$ as (Sarazin, 1988)

$$
M_{\mathrm{G}}(R)=-\frac{k T R}{\mu G m_{\mathrm{p}}}\left(\frac{\mathrm{d} \ln p}{\mathrm{~d} \ln R}\right),
$$

where $G$ is the constant of gravity, $k$ is the Boltzmann constant, $T$ is the ICM temperature, $\mu \sim 0.6$ is the mean particle mass in unit of the proton mass $m_{\mathrm{p}}$, and $p$ is the ICM pressure. When integrated up to $R \sim 1 \mathrm{Mpc}\left(=3.08 \times 10^{22} \mathrm{~m}\right)$, a typical cluster has $M_{\mathrm{G}}(R)=10^{14 \sim 15} M_{\odot}$, of which $\sim 90 \%$ is carried by the DM, 7\% by the ICM, and only $\sim 3 \%$ by the stellar component.

\section{Properties of the ICM}

\subsection{Basic parameters of the ICM}

The ICM has a typical temperature of $2-12 \mathrm{keV}$, presumably heated by adiabatic compression when the cluster formed. The ICM consists mainly of primordial gas $(76 \%$ hydrogen and $23 \%$ helium by weight), plus heavier elements (O, Si, Fe etc.) to a level of $0.25-0.3$ solar abundances. Therefore, the ICM contains matter once processed in the stellar interior, and then ejected, by supernovae and other mechanisms. 


\section{Coma Cluster}

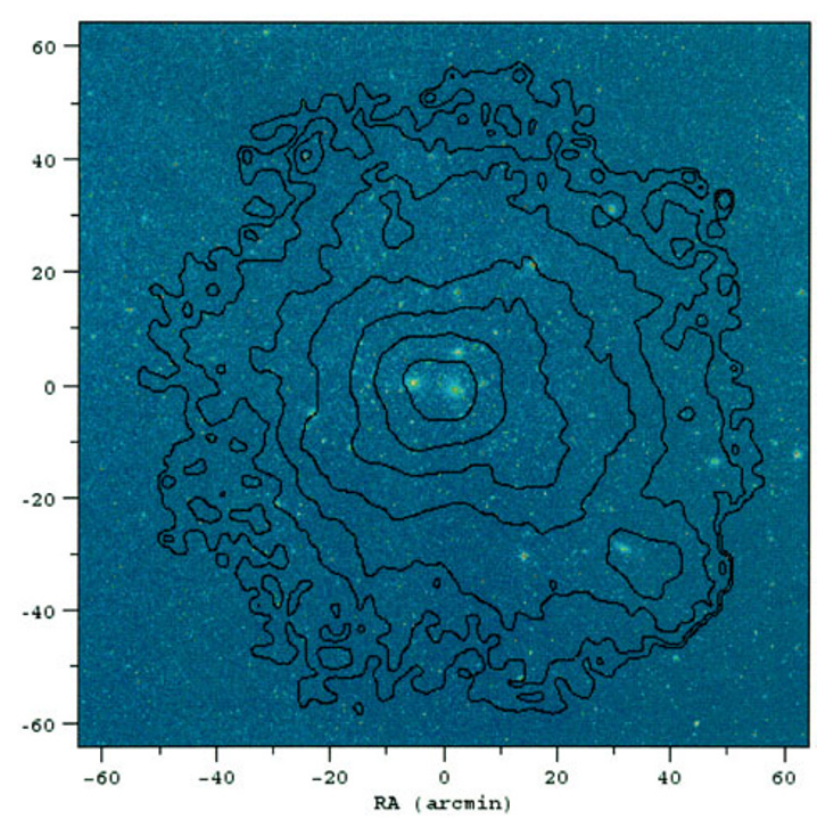

Fig. 1. X-ray surface brightness contours of the Coma cluster of galaxies, superposed on an optical image.

The radial profile of the ICM density is approximated by a Lorenzian-like form as

$$
n(R)=n_{0}\left\{1+(R / a)^{2}\right\}^{-3 \gamma / 2}
$$

where $n_{0}=10^{3 \sim 4} \mathrm{~m}^{-3}$ is the central density, $a=100 \sim 250$ $\mathrm{kpc}$ is a characteristic core radius, and $\gamma$ is an empirical shape parameter which usually takes values of $\sim 2 / 3$. An example of the integrated mass profile of the ICM is given in Fig. 2, together with the total mass profile of Eq. (1) and the stellar mass profile. In this particular case, the integrated ICM mass overwhelms the stellar mass beyond $R \sim 300 \mathrm{kpc}$.

Because of the high temperature and low density, the ICM can be considered as the most ideal, collision-less, fullyionized, classical plasma. Actually, the ratios among the inter-particle separation, the Debye length, the ion Larmor radius (for a magnetic field of $0.1 \mathrm{nT}$ ), the mean free path due to classical Coulomb scattering, and the system size $(\sim 1$ $\mathrm{Mpc})$, become approximately $1: 10^{3}: 10^{9.5}: 10^{19}: 10^{22.5}$.

\subsection{The ICM magnetization}

Like most of the other astrophysical plasmas, the ICM is generally magnetized (Kronberg, 1994). According to measurements of the Faraday rotation of active galaxies in clusters and background quasars, the magnetic field averaged along the line of sight is typically $\langle B\rangle=0.1 \sim 1 \mathrm{nT}$ (Ge and Owen, 1994). Diffuse synchrotron radio emission observed from a limited number of clusters gives an additional support to the ICM magnetization.

The nominal equipartition field strength in the cluster central regions is $\sim 3 \mathrm{nT}$, so that the measured $\langle B\rangle$ implies a considerably high- $\beta(10 \sim 100)$ situation. However, the field lines may be extremely tangled, in which case $\langle B\rangle$ significantly underestimates the local field strength. We presume the local field strength could be as strong as the equiparti-

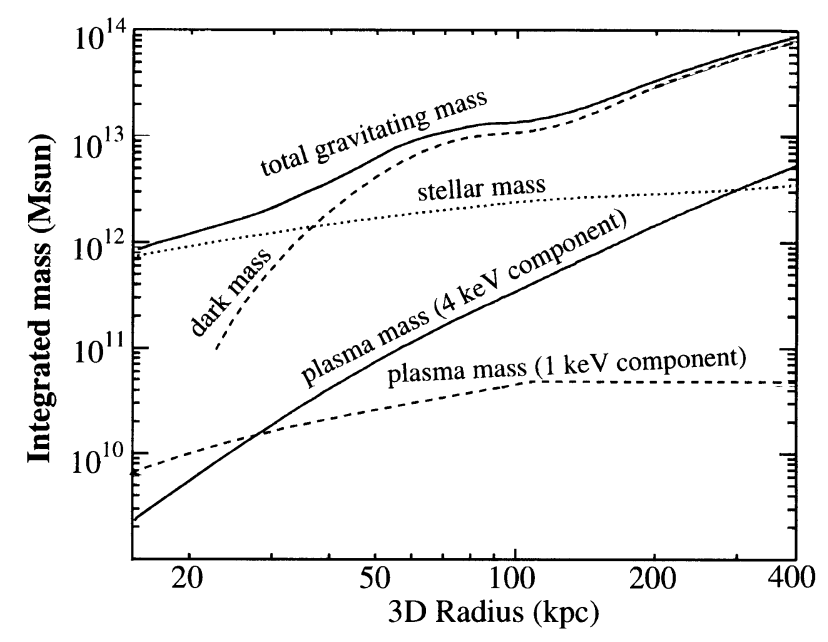

Fig. 2. Radially integrated profiles of various mass components of the Centaurus cluster (Ikebe et al., 1999); the total mass (upper solid curve), the stellar mass (dotted curve), the hot ICM mass (lower solid curve), and the cool plasma mass (lower dashed curve). The upper dashed curve represents the DM mass, obtained by subtracting the three baryonic components from the total mass.

tion value. In any case, the magnetic Reynolds number is absurdly high.

\subsection{Dynamical and thermal state of the ICM}

Since the ICM is hydrostatically confined by gravity in a spherical potential, we expect it to be in the condition of minimum free energy, and hence to suffer from none of major MHD instabilities (interchange-type ones, current-driven ones, etc.). Since the radiative cooling time of the ICM

$$
t_{\mathrm{c}}=2 \times 10^{9}\left(\frac{T}{4 \mathrm{keV}}\right)^{-1 / 2}\left(\frac{n}{10^{4} \mathrm{~m}^{-3}}\right)^{-1} \mathrm{yr}
$$

is typically $\sim 1 / 6$ of the Hubble time, the ICM near the cluster center would gradually lose energy via X-ray radiation, causing a slow radial in-flow of the ICM to compensate the radiative energy loss. This idea, called cooling flow, has been developed as a popular account of X-ray properties of clusters (Fabian, 1994).

The cooling-flow hypothesis, however, ignores an important fact: a large number of member galaxies are actually moving through the ICM, with a velocity of $300 \sim 1000$ $\mathrm{km} \mathrm{s}^{-1}$ which is close to the ICM sound speed. (That is, in each cluster, the galaxies and the ICM have comparable energies per unit mass.) Astrophysicists would think that the galaxies swim through the ICM without much interaction, since the galaxy motion is at most transonic. However, plasma physicists would think otherwise, since the galaxies, having their own inter-stellar plasmas and inter-stellar magnetic fields, must be "opaque" to the ICM in the MHD sense. Therefore, the galaxy motion is expected to strongly "drag" the ICM, causing intense MHD turbulence where frequent magnetic reconnection should occur. Possible "wake" from such an interaction has recently been detected in X-rays by Drake et al. (2000). As a result, kinetic energy of the galaxies will be dissipated efficiently into the ICM heating and particle acceleration. More detailed aspects of such processes are described in Section 4. 


\section{Evidence for Galaxy-ICM Interaction}

Based mainly on X-ray observations of clusters of galaxies using ASCA launched in 1993 (Tanaka et al., 1994), we have obtained a number of new results which can be understood as a consequence of MHD interactions between the moving galaxies and the ICM (Makishima, 1996, 1997a,b, 1999a,b).

\subsection{Two-phase picture of the ICM}

Although the ICM is relatively isothermal in outer cluster regions, its temperature has long been known to decrease markedly toward the center of many clusters. This was so far interpreted as a result of ongoing ICM cooling (i.e., the cooling flow), because of the short cooling time of Eq. (3).

With $A S C A$, we have confirmed the presence of $\operatorname{cool}(\sim 1$ $\mathrm{keV}$ ) emission components within $\sim 100 \mathrm{kpc}$ of the center in many clusters of galaxies (e.g. Fabian et al., 1994). However, what we have actually observed is somewhat different from the prediction of the cooling-flow hypothesis. The plasma has been found to consist mainly of two (hot and cool) components (Fukazawa et al., 1994) as exemplified in Fig. 3, rather than displaying a continuous temperature distribution characteristic of ongoing cooling. The central volume appears to be occupied predominantly by the hot component, where the cool component is somehow mixed in (Fukazawa et al. 1994; Ikebe et al., 1997, 1999), forming a "two-phase" region. The cool component is clearly associated with the "cD" galaxy (Fukazawa et al., 2000), the dominant elliptical galaxy found at the center of about two-thirds of clus-

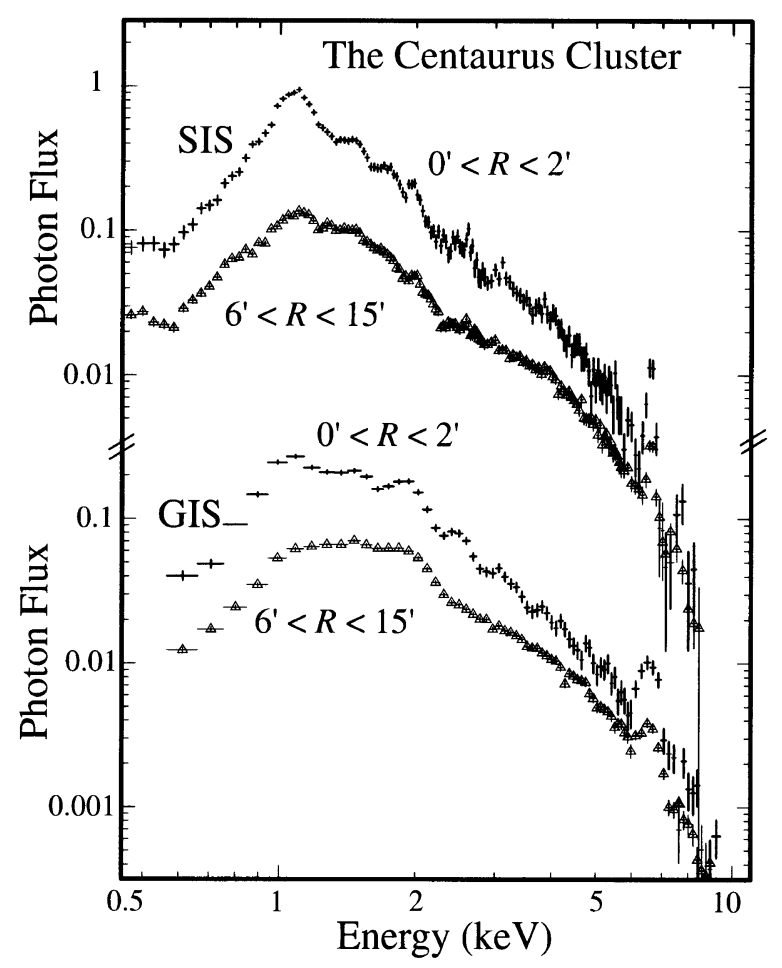

Fig. 3. The X-ray spectra of the Centaurus cluster (Fukazawa et al., 2000) obtained with the two instruments (SIS and GIS) onboard ASCA. Triangles represent the spectra taken from the outer cluster region, where an isothermal $(\sim 4 \mathrm{keV}) \mathrm{ICM}$ produces Fe-K emission line at $6.6 \mathrm{keV}$. Crosses show the spectra from the central $100 \mathrm{kpc}$ region, where an additional cool $(\sim 1 \mathrm{keV})$ component gives rise to $\mathrm{S}-\mathrm{K}($ at $\sim 2.5 \mathrm{keV}), \mathrm{Si}-\mathrm{K}$ (at $\sim 1.9 \mathrm{keV})$, and $\mathrm{Mg}-\mathrm{K}$ (at $\sim 1.4 \mathrm{keV}$ ) lines, as well as the strong Fe-L complex over the $0.7-1.2 \mathrm{keV}$ region. ters; clusters without a $\mathrm{cD}$ galaxy lack the cool component (Tamura et al., 2000). The central two-phase region exhibits a considerably higher metallicity and a slightly higher $\mathrm{Fe} / \mathrm{Si}$ ratio than the bulk of the ICM, suggesting a significant influence from Type I supernovae in the $\mathrm{cD}$ galaxy (Fukazawa et $a l ., 2000)$. Furthermore, we have discovered a central "dimple" in the gravitational potential around each cD galaxy $(\mathrm{Xu}$ et al., 1998), which is suspected to have caused overestimates of the cooling-flow rate.

Although we have thus arrived at a steady-state two-phase picture instead of the cooling-flow scenario, we must explain how the two phases are thermally insulated from each other, and what prevents the cool phase from the radiative collapse. For this purpose, we assume that the MHD turbulence produces chaotic magnetic fields all over the cluster volume, except at the very center where the $\mathrm{cD}$ galaxy is standstill with respect to the ICM. Then, a well-ordered magnetic corona will develop around each $\mathrm{cD}$ galaxy, with many closed magnetic loops anchored to it. We assume the loop interior to be filled with the cool phase originating from the cD galaxy, whereas the exterior is permeated by the hot ICM (Makishima, 1997a,b, 1999a,b). Thus, the two phases can be thermally decoupled.

\subsection{ICM heating by the galaxy motion}

In order for the cool phase to be sustained for nearly the Hubble time, it must be heated by some mechanisms, against its radiative energy loss with a typical luminosity of $10^{35.5 \sim 36.5} \mathrm{~W}$. We speculate that the motion of other galaxies around the $\mathrm{cD}$ galaxy will generate intense MHD turbulence around the central corona, wherein frequent magnetic reconnection heats the ICM.

Then, is the proposed mechanism efficient enough? For a typical cluster containing $\sim 100$ galaxies with a velocity dispersion of $v=700 \mathrm{~km} \mathrm{~s}^{-1}$, the ICM heating luminosity due to the galaxy drag becomes $L_{\text {drag }} \sim 100 \xi n m_{\mathrm{p}} v^{3} S \sim$ $2 \times 10^{38} \xi \mathrm{W}$ (Makishima, 1999a,b). Here $\xi$ is the efficiency of thermalization, $n \sim 3 \times 10^{3} \mathrm{~m}^{-3}$ is the ICM density, and $S=\pi r^{2}$ with $r \sim 20 \mathrm{kpc}$ is the MHD cross section of each galaxy. Even if $\xi$ is moderately low, e.g. $\sim 0.05$, we expect $L_{\text {drag }} \sim 1 \times 10^{37} \mathrm{~W}$. Further assuming a total stellar mass of $1 \times 10^{13} M_{\odot}$, the total kinetic energy in galaxies, $5 \times 10^{54} \mathrm{~J}$, can sustain the heating for $\sim 1.6 \times 10^{10} \mathrm{yr}$, i.e. over the Hubble time.

Invoking a loop-like geometry has another merit, that the heating and cooling can be balanced in a stable manner, via a mechanism analogous to the Rosner-Tucker-Vaiana (1978) self-regulating mechanism which is operating in the solar corona (Kano and Tsuneta, 1995). That is, if the heating rate due to reconnection becomes too low, the loops become thinner, compressed by the external pressure, and the conductive flux automatically reduces to recover the thermal balance.

\subsection{Reaction to the galaxies}

If the ICM is actually exerting friction to the moving galaxies, we should expect them to receive significant reactions from the ICM. In fact, we find several pieces of evidence supporting this idea.

As is clear from Fig. 2, the galaxy distribution is generally more concentrated than the ICM mass profile; within $\sim 100$ $\mathrm{kpc}$, the ICM mass is only $\sim 10 \%$ of the stellar mass. Although we cannot rule out the possibility that the efficiency 
of galaxy formation was lower in outer cluster regions, it may be explained alternatively as a manifestation of gradual galaxy infall to the potential bottom, as a consequence of the ICM friction. In other words, the galaxies are inferred to have lost their kinetic energies, which in turn were deposited onto the ICM to make it more extended.

The proposed interaction with the ICM will also accelerate mergers between a pair of close-by galaxies, because their orbital angular momentum can be dumped on the ICM by launching helical Alfvén waves. We hence expect a dense ICM environment to enhance the mergers of spiral galaxies into bigger and fewer elliptical galaxies. Actually, it is well known that the fraction of spiral galaxies rapidly decreases to the cluster center (e.g. Whitmore and Gilmore, 1993). Ramirez and de Souza (1998) report that orbits of elliptical galaxies in clusters tend to be radial, while those of spirals are rather circular so that they avoid the central region during their journey. Furthermore, as to galaxy groups (which are the poorest of clusters of galaxies), X-ray luminous (i.e. plasma-rich) systems have been found to contain a higher fraction of elliptical galaxies (Mulchaey et al., 1996). All these facts support our conjecture.

Another important fact from the evolutionary viewpoint is that distant clusters contain a large number of small, disturbed, blue spiral galaxies, while nearby ones do not (Dressler et al., 1994). We can interpret this finding that the spiral galaxies in the past have merged into ellipticals, through the enhanced galaxy-galaxy interaction aided by the ICM. This mechanism can be much more efficient than the simple gravitational close encounter proposed by Moore et al. (1996).

\subsection{Particle acceleration}

Yet another possible consequence of the proposed galaxy vs. ICM interaction is particle acceleration by the magnetic reconnection. In the vast intracluster space, the particles are expected to attain very high energies because of rather low energy loss rate there. Actually, according to an empirical scaling law proposed by Makishima (1999b), the maximum particle energy attainable in an acceleration site is roughly given by $E_{\max }=v \times B \times L$, where $v$ is the typical internal velocity of the system, $B$ is again the magnetic field, and $L$ is the system size. Substituting $v=7 \times 10^{5} \mathrm{~m} \mathrm{~s}^{-1}, B=1 \mathrm{nT}$, and $L=1 \mathrm{Mpc}$, we obtain $E_{\max } \sim 10^{19} \mathrm{eV}$, which is close to the energy of the highest-energy cosmic rays (Takeda et al., 1998).

The reduced energy-loss rate, on the other hand, implies a difficulty of detecting such energetic particles. However, there are increasing number of detections of non-thermal radiation from clusters and groups of galaxies, which signals the presence of energetic particles. One is the excess hard Xrays detected with BeppoSAX from several clusters (FuscoFemiano et al., 1999; Kaastra et al., 1999), which may be inverse-Compton photons or non-thermal Bremsstrahlung radiation (Sarazin and Kempner, 2000). Another is excess soft X-ray signal detected with EUVE from several clusters (e.g., Sarazin and Lieu, 1998), interpreted as an inverseComptonization of the cosmic microwave photons, although thermal interpretation is still possible (Lieu et al., 1999). The other is clear hard X-ray tail, observed with ASCA (Fukazawa et al., 2001) from a fair number of galaxy groups, which is interpreted as either the inverse-Compton radiation by extremely relativistic particles or non-thermal Bremsstrahlung by semi-relativistic electrons.

\section{Future Prospect}

In order to strengthen the plasma-physics view of galaxy clusters developed in the present work, a number of future observations in X-rays and $\gamma$-rays can be proposed. One is the high-resolution spectroscopy of the ICM X-rays, where we expect to find broadening of the atomic emission lines to a level of several hundred $\mathrm{km} \mathrm{s}^{-1}$, due to the predicted plasma turbulence. Another is higher-sensitivity search for non-thermal radiation from galaxy clusters and groups, to obtain further insight into the possible particle acceleration. These two observations could have been carried out best with the X-ray Spectrometer (XRS) and the Hard X-ray Detector (HXD; Takahashi et al., 1996) respectively, both onboard the 5th Japanese cosmic X-ray satellite ASTRO-E developed under an extensive Japan-US collaboration. However, to our great regret, ASTRO-E has been lost on 2000 February 10, because of a malfunctioning in the first stage of the M-V-4 launch vehicle. We are currently practicing our best effort to rebuild ASTRO-E together with its onboard instruments, and to launch it on the soonest possible occasion.

Even though ASTRO-E has been lost, we can utilize the other two high-performance X-ray observatories, the American Chandra and the European XMM-Newton. Using Chandra with the unprecedented angular resolution, we may search the ICM surface brightness around $\mathrm{cD}$ galaxies for the proposed filamentary structure. We may also utilize $X M M$ Newton to accurately measure the ICM profiles of distant clusters, and compare the results with the optical galaxy density profiles. If our conjecture on the galaxy infall is correct, we may find an evolutionary effect in the radial galaxy distribution with respect to the X-ray extent.

\section{References}

Drake, N., M. R. Merrifield, I. Sakelliou, and J. C. Pinkney, X-ray wakes in Abell 160, Mon. Not. R. Astr. Soc., 314, 768-774, 2000.

Dressler, A., A. Oemler, Jr., W. B. Sparks, and R. A. Lucas, New images of the distant, rich cluster CL $0939+4713$ with WFPC2, Astrophys. J., 435, L23-L26, 1994.

Fabian, A. C., Cooling flows in clusters of galaxies, Ann. Rev. Astr. Astrophys., 32, 277-318, 1994.

Fabian, A. C., K. A. Arnaud, M. W. Bautz, and Y. Tawara, ASCA observations of cooling flows in clusters of galaxies, Astrophys. J., 436, L63-L66, 1994.

Fukazawa, Y. et al., Metal concentration and X-ray cool spectral component in the central region of the Centaurus cluster of galaxies, Pub. Astr. Soc. Japan, 46, L55-L58, 1994.

Fukazawa, Y. et al., Statistical properties of metal abundances of the intracluster medium in the central region of clusters, Mon. Not. R. Astr. Soc. 313, 21-31, 2000

Fukazawa, Y. et al., Detection of excess hard X-ray emission from the group of galaxies HCG 62, Astrophys. J. Lett., 547, L119-L122, 2001.

Fusco-Femiano, R. et al., Hard X-ray radiation in the Coma cluster spectrum, Astrophys. J., 513, L21-L24, 1999.

Ge, J. and F. N. Owen, Faraday rotation in cooling flow clusters of galaxies 2: Survey, Astron. J., 108, 1523-1533, 1994

Ikebe, Y. et al., Structure of the X-Ray-emitting gas in the Hydra A cluster of galaxies, Astrophys. J., 481, 660-672, 1997.

Ikebe, Y. et al., Two-phase intracluster medium in the Centaurus cluster of galaxies, Astrophys. J., 525, 58-79, 1999.

Kaastra, J. S. et al., High- and low-energy nonthermal X-ray emission from the Abell 2199 cluster of galaxies, Astrophys. J., 519, L119-L122, 1999. 
Kano, R. and S. Tsuneta, Scaling law of solar coronal loops obtained with Yohkoh, Astrophys. J., 454, 934-944, 1995.

Kronberg, P. P., Extragalactic magnetic fields, Rep. Prog. Phys., 325-382, 1994.

Lieu, R., M. Bonamente, and J. P. D. Mittaz, Extreme-ultraviolet images of the clusters of galaxies A2199 and A1795, Astrophys. J., 517, L91-L95, 1999.

Makishima, K., X-ray probing of the galaxy/cluster interface, in $U V$ and $X$-ray Spectroscopy of Astrophysical and Laboratory Plasmas, edited by K. Yamashita and T. Watanabe, pp. 167-173, Universal Academy Press, Tokyo, 1996.

Makishima, K., Magnetohydrodynamic interpretations of high-energy phenomena in galaxies and clusters, Plasma Phys. Control. Fusion, 39, A15A21, 1997a.

Makishima, K., Magnetohydrodynamic (MHD) view of plasmas in galaxies and clusters, in Imaging and Spectroscopy of Cosmic Hot Plasmas, edited by F. Makino and K. Mitsuda, pp. 137-148, Universal Academy Press, Tokyo, 1997b.

Makishima, K., From the sun to the galaxy clusters, in Observational Plasma Astrophysics: Five Years of Yohkoh and Beyond, edited by T. Watanabe, T. Kosugi, and A. Sterling, pp. 51-60, Kluwer Academic, Dordrecht, 1999a.

Makishima, K., Energy non-equipartition processes in the Universe, Astronomische Nachrichten, 320, 161-166, 1999b.

Moore, B., N. Katz, G. Lake, A. Dressler, and A. Oemler, Jr., Galaxy harassment and the evolution of clusters of galaxies, Nature, 379, 613616, 1996.

Mulchaey, J., D. Davis, R. Mushotzky, and D. Burstein, The intragroup medium in poor groups of galaxies, Astrophys. J., 456, 80-97, 1996.

Ramirez, A. and R. E. de Souza, Shape of the galactic orbits in clusters, Astrophys. J., 496, 693-705, 1998.

Rosner, R., W. Tucker, and G. Vaiana, Dynamics of the quiescent solar corona, Astrophys. J., 643, 643-665, 1978.

Sarazin, C. L., X-Ray Emission from Clusters of Galaxies, Cambridge University Press, 1988

Sarazin, C. L. and J. Kempner, Nonthermal bremsstrahlung and hard X-ray emission from clusters of galaxies, Astrophys. J., 533, 73-83, 2000.

Sarazin, C. L. and R. Lieu, Extreme-ultraviolet emission from clusters of galaxies, Astrophys. J., 494, L177-L180, 1998.

Takahashi, T. et al., Development of the Hard X-ray Detector for the ASTRO-E mission, Astron. Astrophys. Suppl., 120, 645-648, 1996.

Takeda, M. et al., Extension of the cosmic-ray energy spectrum beyond the predicted GZK cutoff, Phys. Rev. Lett., 81, 1163-1166, 1998.

Tamura, T., K. Makishima, Y. Fukazawa, Y. Ikebe, and H. Xu, X-ray measurements of the gravitational potential profile in the central region of the Abell 1060 cluster of galaxies, Astrophys. J., 535, 602-614, 2000.

Tanaka, Y., H. Inoue, and S. S. Holt, The X-ray astronomy satellite ASCA, Pub. Astr. Soc. Japan, 46, L37-L39, 1994.

Whitmore, B. C. and D. M. Gilmore, What determines the morphological fractions in clusters of galaxies?, Astrophys. J., 407, 489-509, 1993.

Xu, H., K. Makishima, Y. Fukazawa, Y. Ikebe, K. Kikuchi, T. Ohashi, and T. Tamura, Discovery of the central excess brightness in hard X-rays in the cluster of galaxies Abell 1795, Astrophys. J., 500, 738-749, 1998.

K. Makishima (e-mail: maxima@phys.s.u-tokyo.ac.jp) 